\title{
Bader's Theory of Atoms in Molecules (AIM) and its Applications to Chemical Bonding
}

\author{
P SHYAM VINOD KUMAR, V RAGHAVENDRA and V SUBRAMANIAN* \\ Chemical Laboratory, CSIR-Central Leather Research Institute, Adyar, Chennai, Tamil Nadu 600 020, India \\ e-mail: subuchem@hotmail.com; subbu@clri.res.in
}

MS received 22 July 2016; revised 17 August 2016; accepted 19 August 2016

\begin{abstract}
In this perspective article, the basic theory and applications of the "Quantum Theory of Atoms in Molecules" have been presented with examples from different categories of weak and hydrogen bonded molecular systems.
\end{abstract}

Keywords. QTAIM; non-covalent interaction; chemical bonding; H-bonding; electron density

\section{Introduction}

It is now possible to define the structure of molecules quantum mechanically with the help of Bader's Quantum Theory of Atoms in Molecules (QTAIM). ${ }^{1,2}$ This theory has been widely applied to unravel atom-atom interactions in covalent and non-covalent interactions in molecules, ${ }^{3}$ molecular clusters, ${ }^{4}$ small molecular crystals, ${ }^{5}$ proteins, ${ }^{6}$ DNA base pairing and stacking. ${ }^{7}$ Basic motive of QTAIM is to exploit charge density or electron density of molecules $\rho(\mathbf{r} ; \mathbf{X})$ as a vehicle to study the nature of bonding in molecular systems. The electron density (ED) in general can be defined as, ${ }^{2,8,9}$

$$
\rho(\mathbf{r} ; \mathbf{X})=N \int d \tau^{\prime} \psi^{*}(\mathbf{x} ; \mathbf{X}) \psi(\mathbf{x} ; \mathbf{X})
$$

where $\mathrm{N}$ is the no. of electrons, $\mathrm{x}$ is the electronic coordinates, $\mathrm{X}$ is the nuclear coordinates and $d \tau^{\prime}$ represents the volume element of the system under consideration.

The ED distribution is predominantly affected by the interaction between two nuclei and hence the chemical bonding. It is found from previous reports that both theoretical ${ }^{4}$ and experimental ${ }^{5}$ electron densities of molecules can be used to gain insight into the nature of the chemical bonding. In fact, the topological analysis of ED distributions has expanded beyond the territories of theoretical chemistry to the X-ray crystallography. The topological properties of ED and its derivatives are found to be immensely useful in delineating the concept of the bonding through bond paths and bond critical points (BCPs). ${ }^{2,3}$ The molecular graph and the ED along with its derivatives at the $\mathrm{BCPs}$ provide a precise

*For correspondence

Celebrating 100 years of Lewis Chemical Bond account of the bonding within a molecule or crystal. Specially, the derivative of electron density on BCP is zero. Bader's group has made several seminal contributions to the development of the QTAIM theory and its applications to unravel chemical bonding. ${ }^{10-13}$ Popelier and coworkers have employed the QTAIM to address several issues in chemistry. ${ }^{14-16}$ Particularly, they have demonstrated the possibility of developing structure-activity-relationship to predict various physico-chemical properties. ${ }^{17}$ They have used the theory of Quantum Chemical Topology (QCT), to provide $a b$ initio descriptors that are able to accurately predict pKa values for 228 carboxylic acids. ${ }^{18,19}$ Hydrogenbond strengths have been described in terms of basicities with the help of several different scales. Bohorquez, Boyd, and Matta have illustrated the concept of deriving molecular model with quantum chemical bonding with the help of the theory of QTAIM. ${ }^{1}$ Matta and coworkers have characterized the tri-hydrogen bond based on topology of ED. ${ }^{20}$

Several groups have applied the QTAIM for unravelling the non-covalent interactions such as weak van der Waals, $\pi \ldots \pi, \mathrm{X}-\mathrm{H} . . \pi$, conventional hydrogen bonding, cation... $\pi$ interactions, halogen bonds, etc. and also other applications in chemistry. ${ }^{3,21-24}$ All these interactions have been vividly characterized with the help of theory of QTAIM. It is not overstating that QTAIM has completely provided new dimensions to the concept of hydrogen bonding. Grabowski and coworkers have made several significant contributions to the characterization and understanding of hydrogen bonding interactions in various systems with the help of QTAIM. ${ }^{25,26}$ It is evident from previous studies that Bader's topological parameters exhibited interrelationship with geometrical parameters. For example, the 
interrelationship between atom-atom distance and the ED at the BCP has been investigated. ${ }^{3}$ This relationship revealed that $\mathrm{ED}$ at the $\mathrm{BCP}$ provides a good measure of strength of interaction between two molecular systems. It is also important to mention that QTAIM analysis provided clear description of degree of covalency in the hydrogen bonding interaction. Espinosa and coworkers ${ }^{27,28}$ have used parameters derived from the QTAIM analysis to elicit degree of covalency in hydrogen bonding interaction. A measure of the strength $\left(\Delta_{\text {com }}\right)$ of hydrogen bonding interaction has been developed with the help of QTAIM derived topological and geometrical parameters. ${ }^{29}$ It is given as:

$$
\begin{aligned}
\Delta_{c o m}=\{[ & \left.\left(r_{\mathrm{A}-\mathrm{H}}-r_{\mathrm{A}-\mathrm{H}}^{0}\right) / r_{\mathrm{A}-\mathrm{H}}^{0}\right]^{2}+\left[\left(\rho_{\mathrm{A}-\mathrm{H}}^{0}-\rho_{\mathrm{A}-\mathrm{H}}\right) / \rho_{\mathrm{A}-\mathrm{H}}^{0}\right]^{2} \\
& \left.+\left[\left(\nabla^{2} \rho_{\mathrm{A}-\mathrm{H}}-\nabla^{2} \rho_{\mathrm{A}-\mathrm{H}}^{0}\right) / \nabla^{2} \rho_{\mathrm{A}-\mathrm{H}}^{0}\right]^{2}\right\}^{\frac{1}{2}}
\end{aligned}
$$

where $r_{\mathrm{A}-\mathrm{H}}, \rho_{\mathrm{A}-\mathrm{H}}$ and $\nabla^{2} \rho_{\mathrm{A}-\mathrm{H}}$ represent proton donating bond involved in $\mathrm{H}$-bonding, the bond length, electronic density at A-H bond critical point and Laplacian of this density respectively. $r_{\mathrm{A}-\mathrm{H}}^{0}, \rho_{\mathrm{A}-\mathrm{H}}^{0}$ and $\nabla^{2} \rho_{\mathrm{A}-\mathrm{H}}^{0}$ correspond to the same parameters of the $\mathrm{A}-\mathrm{H}$ bond involved in H-bond formation or any other nonbonded interaction.

This complex parameter clearly describes the covalency of hydrogen bonding interaction. Higher values of $\Delta_{\text {com }}$ indicate lengthening of the proton donating bond and shorter proton-acceptor distances. Higher value of $\Delta_{\text {com }}$ implies greater binding energy and negative value of the total electron energy density at hydrogen bond critical point(HBCP) and hence the degree of covalency of hydrogen bonding interaction. In addition to QTAIM, electron delocalization function (ELF), Natural Bond Orbital analysis (NBO) and energy decomposition analysis (EDA) are also useful in describing the covalency of hydrogen bonding interaction. ${ }^{2}$ In understanding degree of covalency of hydrogen bonding interaction, Parthasarthi et al., have shown that there is a linear relationship between interaction energy (IE) and $\rho\left(\mathbf{r}_{\mathbf{c}}\right)$ at the BCP for diverse class of molecular systems. ${ }^{30}$ It is also evident from that report that weak, moderate, strong and very strong hydrogen bonds can be clearly described with the help of ED and its Laplacian at BCPs. The relationship between Stabilization Energy (SE) and ED at BCP explain the smooth transition in the hydrogen strength from van der Waals to covalent limit.

The detailed theory of atoms in molecules has been explained in the Bader's Book. ${ }^{2 a}$ Other monographs on the QTAIM also provide basic theory and its applications. ${ }^{31,32}$ In this perspective article, the basic theory and applications of QTAIM in eliciting the weak, van der Waals, hydrogen bonding and ionic bonding interactions in various molecules and molecular clusters have been described with suitable examples. Since voluminous information is available on this topic, the comprehensive coverage of theory and applications of QTAIM is beyond the scope of this perspective article. However, important aspects of the theory are provided in this article.

\subsection{The basic theory of quantum theory of atoms in molecule}

It is well known that the ED distribution of a molecular system is the physical manifestation of the forces acting within the molecular system. ${ }^{2}$ The attractive force exerted by the nuclei determines the most important topological properties of ED of multi-electronic system. The ED exhibits local maxima only at the positions of the nuclei. Exceptions to this have also been observed under certain circumstances. It is possible to recognize atomic forms within the molecules with the help of local maxima exhibited by the ED at the nuclei. The ED distribution, ED relief map and molecular graph of benzene are shown in Figure 1, which clearly reveal the presence of local maxima at the position of nucleus.

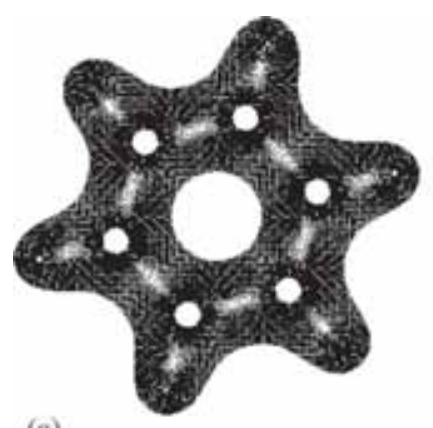

(a)

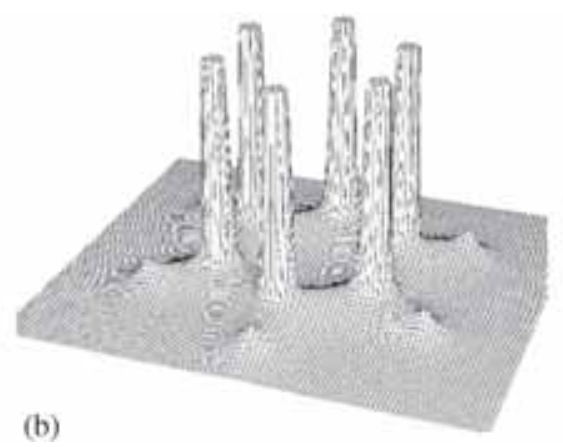

(b)

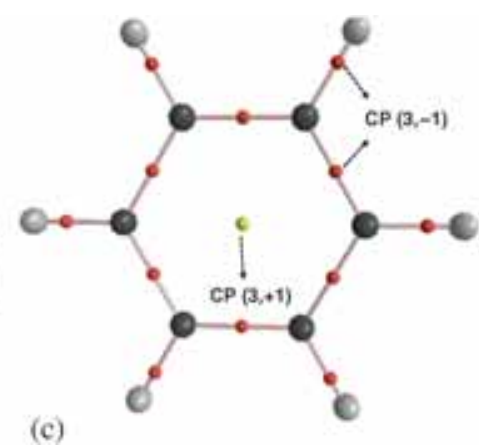

(c)

Figure 1. (a) Electron density contour map; (b) Relief map; (c) Molecular graph of Benzene. 


\subsection{Topography of electron density distribution and critical points}

It can be inferred from Figure 1 that the ED exhibits a maximum, a minimum, or a saddle point in space. These special points are referred to as Critical Points (CPs). At this point, the first derivatives of $\rho\left(\mathbf{r}_{\mathbf{c}}\right)$ vanishes, i.e., $\nabla \rho\left(\mathbf{r}_{\mathbf{c}}\right)=0$, where $\nabla \rho\left(\mathbf{r}_{\mathbf{c}}\right)$ is given in equation (3) and $\mathbf{r}_{\mathbf{c}}$ is the $\mathrm{CP}$.

$$
\nabla \rho\left(\mathbf{r}_{\mathbf{c}}\right)=\mathbf{i} \frac{\partial \rho}{\partial x}+\mathbf{j} \frac{\partial \rho}{\partial y}+\mathbf{k} \frac{\partial \rho}{\partial z}
$$

It is well known that a maximum or a minimum or an extremum is determined by the sign of its second derivative at this point. Therefore, it is necessary to explore the second order derivatives of ED. For an arbitrary choice of coordinate axes, nine second order derivatives are possible. It is represented in the form of a real and symmetric matrix known as the Hessian of $\rho\left(\mathbf{r}_{\mathbf{c}}\right)$. It can be diagonalized with the help of unitary transformation to obtain eigenvalues; $\lambda_{1}, \lambda_{2}$ and $\lambda_{3}$ are the principal axes of curvature because the magnitude of the three second derivatives of $\rho\left(\mathbf{r}_{\mathbf{c}}\right)$ calculated with respect to these axes are extremized.

$$
\nabla^{2} \rho\left(\mathbf{r}_{\mathbf{c}}\right)=\frac{\partial^{2} \rho\left(\mathbf{r}_{\mathbf{c}}\right)}{d x^{\prime 2}}+\frac{\partial^{2} \rho\left(\mathbf{r}_{\mathbf{c}}\right)}{d y^{\prime 2}}+\frac{\partial^{2} \rho\left(\mathbf{r}_{\mathbf{c}}\right)}{d z^{\prime 2}}=\lambda_{1}+\lambda_{2}+\lambda_{3}
$$

The CPs are designated as $(\omega, \sigma)$, where $\omega$ is rank of $\mathrm{CP}$ and $\sigma$ is its signature. The rank of a $\mathrm{CP}$ is equal to the number of non-zero eigenvalues (non-zero curvatures of $\rho\left(\mathbf{r}_{\mathbf{c}}\right)$ at the CPs) and signature is the algebraic sum of the signs of eigenvalues (signs of curvatures of $\rho\left(\mathbf{r}_{\mathbf{c}}\right)$ at the CPs) There are four possible values for CPs of rank three. They are $(3,-3),(3,-1),(3,+1)$ and $(3,+3)$. In the case of $(3,-3)$, all curvatures are negative and $\rho$ is a local maximum at $\mathbf{r}_{c}$. For $(3,-1)$, the two curvatures are negative and $\rho$ is a maximum at $\mathbf{r}_{c}$ is in the plane defined by their corresponding axes. Further, $\rho$ is a minimum at $\mathrm{CP}$ along the third axis, which is perpendicular to this plane. The $(3,+1) \mathrm{CP}$ has two positive curvatures and $\rho$ is a minimum at $\mathrm{CP}$ in the plane defined by their corresponding axes. Further, $\rho$ is a maximum at $\mathrm{CP}$ along the third axis, which is perpendicular to this plane. The $(3,+3) \mathrm{CP}$ represents all curvatures are positive and $\rho$ is a local minimum at $\mathbf{r}_{c}$.

It is evident from the topological distribution of ED of benzene that $(3,-3) \mathrm{CP}$ occurs at the nuclear positions. In the QTAIM parlance, the nuclei act as the attractors of the gradient of ED distribution of molecules. The basin of attractor is a region of threedimensional space, which extends throughout all the space. An atom is defined as the union of attractor and its associated basin. ${ }^{2}$ It can be seen from Figure 1c that $(3,-1) \mathrm{CP}$ is found between every pair of nuclei which are linked by a chemical bond in the benzene molecule. It is found from detailed analysis of topological features of ED that pairs of gradient paths which originate at each $(3,-1) \mathrm{CP}$ and terminate at the neighboring attractors. ${ }^{2}$ These two unique paths describe a line through the charge distribution connecting neighboring nuclei along which $\rho$ is a maximum with respect to any neighboring line. Such a line is known as atomic interaction line in the topological analysis of ED distribution. ${ }^{2}$ The presence of a $(3,-1) \mathrm{CP}$ and related atomic interaction line highlights that electronic charge density is accumulated between the nuclei that are bonded. The existence of an atomic interaction line in an equilibrium geometry satisfies both necessary and sufficient conditions that the atoms be bonded to one another. Hence, it is called as bond path and the $(3,-1)$ $\mathrm{CP}$ is referred to as bond critical point (BCP). All these topological features define molecular graph.

Other CPs of rank three arise due to the particular geometrical arrangements of bond paths. In the case of benzene, the bond paths are connected to form a ring. The $(3,+1) \mathrm{CP}$ found in the interior of the ring is known as ring critical point (RCP). A ring is defined as a part of a molecular graph, which bounds a ring surface. ${ }^{2}$ If the bond paths enclose the interior of a molecule with ring surfaces, then the Cage Critical Point (CCP) $(3,+3)$ is observed. ${ }^{2}$

\subsection{Laplacian of electron density}

In the topological analysis of ED, the Laplacian $\left(\nabla^{2} \rho\right)$ plays a very important role in the characterization of chemical bonding. In fact, the $\nabla^{2} \rho$ provides physical basis for the celebrated electron pair model of Lewis. ${ }^{33,34}$ It can be combined with other important concepts in electronic structure theory of molecules. For example, $\nabla^{2} \rho$ along with the electrostatic Hellmann-Feynman theorem facilitates the characterization of binding or non-binding with respect to a given interaction in a molecule. ${ }^{2}$ The $\nabla^{2} \rho\left(\mathbf{r}_{\mathbf{c}}\right)<0$ indicates the concentration of charge towards interaction line. The concentration of charge leads to contraction of $\rho\left(\mathbf{r}_{\mathbf{c}}\right)$ perpendicular to the interaction line and lowers the potential energy. The magnitude of lowering of the potential energy is greater than the kinetic energy from the same region thereby creating attractive force and bound shared interaction. The $\nabla^{2} \rho\left(\mathbf{r}_{\mathbf{c}}\right)>0$ implies that the interaction is dominated by the contraction of $\rho\left(\mathbf{r}_{\mathbf{c}}\right)$ towards each nucleus. The parallel gradient and the curvature of $\rho\left(\mathbf{r}_{\mathbf{c}}\right)$ are large. In this case net forces of repulsion act on the nuclei. 
It is evident from literature that there are other important relationships between energetic topological parameters and the $\nabla^{2} \rho\left(\mathbf{r}_{\mathbf{c}}\right)$ at CPs. One of the important relationships is the local form of virial theorem: ${ }^{2}$

$$
\begin{gathered}
\frac{1}{4} \nabla^{2} \rho\left(\mathbf{r}_{\mathbf{c}}\right)=2 G\left(\mathbf{r}_{\mathbf{c}}\right)+V\left(\mathbf{r}_{\mathbf{c}}\right) \\
H\left(\mathbf{r}_{\mathbf{c}}\right)=V\left(\mathbf{r}_{\mathbf{c}}\right)+G\left(\mathbf{r}_{\mathbf{c}}\right)
\end{gathered}
$$

where $G\left(\mathbf{r}_{\mathbf{c}}\right), V\left(\mathbf{r}_{\mathbf{c}}\right)$, and $H\left(\mathbf{r}_{\mathbf{c}}\right)$ are the kinetic energy, potential energy, and the total electron energy densities respectively. $G\left(\mathbf{r}_{\mathbf{c}}\right)$ is a positive quantity and $V\left(\mathbf{r}_{\mathbf{c}}\right)$ is a negative quantity. The balance between the kinetic electron energy density $G\left(\mathbf{r}_{\mathbf{c}}\right)$ and the potential electron energy density $V\left(\mathbf{r}_{\mathbf{c}}\right)$ reveals the nature of the interaction. If $\left|V\left(\mathbf{r}_{\mathbf{c}}\right)\right|>2 \mathrm{G}\left(\mathbf{r}_{\mathbf{c}}\right)$, then the interaction is covalent in nature. If $\left|V\left(\mathbf{r}_{\mathbf{c}}\right)\right|$ is one time more than the $G\left(\mathbf{r}_{\mathbf{c}}\right)$ then $\nabla^{2} \rho\left(\mathbf{r}_{\mathbf{c}}\right)$ is positive and $H\left(\mathbf{r}_{\mathbf{c}}\right)$ is negative. In this situation, both $\nabla^{2} \rho\left(\mathbf{r}_{\mathbf{c}}\right)$ and $H\left(\mathbf{r}_{\mathbf{c}}\right)$ have been used to characterize bonding interaction. Similarly, the ratio of $-\frac{G\left(\mathbf{r}_{\mathbf{r}}\right)}{V\left(\mathbf{r}_{\mathbf{c}}\right)}$ has also been employed to classify the bonding interaction. ${ }^{35}$ If this ratio is greater than 1 then the nature of the interaction is purely noncovalent. Different criteria for describing the nature of bonding using the topological parameters such as $\rho\left(\mathbf{r}_{\mathbf{c}}\right), \nabla^{2} \rho\left(\mathbf{r}_{\mathbf{c}}\right), V\left(\mathbf{r}_{\mathbf{c}}\right), G\left(\mathbf{r}_{\mathbf{c}}\right)$, and $H\left(\mathbf{r}_{\mathbf{c}}\right)$ have been summarized in previous reports. ${ }^{3,36}$

\subsection{Integrated atomic properties}

The underlying fundamental concept in QTAIM is the topology of ED. The topology of ED determines the natural partitioning of the molecular space into disjoint regions, which are identified as atoms in molecules. ${ }^{37}$ The atoms in molecules are bounded by surface of zeroflux in the gradient vector field of ED as given in the following equation, ${ }^{2}$

$$
\nabla \rho(\mathbf{r}) \cdot \mathbf{n}(\mathbf{r})=0
$$

where, $\nabla \rho(\mathbf{r})$ is the gradient of the electron density and $\mathbf{n}(\mathbf{r})$ is a unit vector normal to the surface. Thus, with the help of atoms in molecule partitioning concept, it is possible to define atomic properties of atoms in molecule. The atomic average of an observable $\hat{A}$ is defined ${ }^{2}$ as

$$
A(\boldsymbol{\Omega}) \equiv\langle\hat{A}\rangle_{\boldsymbol{\Omega}}=\int_{\boldsymbol{\Omega}} d \tau \int d \tau^{\prime}\left(\frac{N}{2}\right)\left\{\psi^{*} \hat{A} \psi+(\hat{A} \psi)^{*} \psi\right\}
$$

where, $\boldsymbol{\Omega}$ is the sub-space, $d \tau$ and $d \tau^{\prime}$ are the volume elements and $N$ is the total number of electrons. The most important outcome of the above definition is the average value of any observable of the molecules, which can be calculated from the ED.
This concept can be used to gain insight into changes in the atomic properties upon bonding with other systems. One of the important properties of atoms in molecule is the net charge on an atom. ${ }^{2}$

$$
\begin{gathered}
N(\boldsymbol{\Omega})=\int_{\boldsymbol{\Omega}} \rho(\mathbf{r}) d \tau \\
q(\boldsymbol{\Omega})=\left(Z_{\boldsymbol{\Omega}}-N(\boldsymbol{\Omega})\right) e
\end{gathered}
$$

where, $N(\boldsymbol{\Omega}), q(\boldsymbol{\Omega})$ and $Z_{\Omega}$ are average number of electrons, net charge on an atom and atomic number respectively.

If A equals the radial distance of an electron from the nucleus, it yields corresponding average over the charge density. Using this definition, the atomic volume $V(\boldsymbol{\Omega})$ can be calculated as a measure of region enclosed by the intersection of its interatomic surfaces and an envelope of charge density of some value. The first moment $\mathbf{M}(\boldsymbol{\Omega})$ of an atom's charge distribution can be obtained from the following equation: ${ }^{2}$

$$
\mathbf{M}(\boldsymbol{\Omega})=-\int_{\boldsymbol{\Omega}} \mathbf{r}_{\boldsymbol{\Omega}} \rho(\mathbf{r}) d \tau
$$

where $\mathbf{r}_{\Omega}$ is vector from the nucleus.

The other important atomic property is quadrupole moment, $Q_{z z}(\boldsymbol{\Omega})$. It is defined as, ${ }^{2}$

$$
Q_{z z}(\boldsymbol{\Omega})=-\int_{\boldsymbol{\Omega}}\left(3 z_{\Omega}^{2}-r_{\boldsymbol{\Omega}}^{2}\right) \rho(\mathbf{r}) d \tau
$$

The changes in these atomic properties after bonding provide finer details on the nature of bonding. Furthermore, it is possible to transfer these atomic or group properties to other systems to develop appropriate relationship. Popelier made very detailed analysis of these properties for hydrogen bonded complexes ${ }^{38,39}$ and added further criteria involving these integrated properties of atoms in molecules. They are, (i) an increase in net charge, (ii) an energetic destabilization, (iii) a decrease in dipolar polarization, and (iv) a decrease in atomic volume for the proper characterization of hydrogen bonding interaction in addition to the correct topological pattern.

\section{Characterization of Chemical Bonds}

Bader and Essen have reported that the hallmark of "shared (covalent)" interactions is the high value of the ED at BCP of order $>10^{-1}$ a.u. and negative value of $\nabla^{2} \rho\left(\mathbf{r}_{\mathbf{c}}\right) .{ }^{12 a}$ The negative value of $\nabla^{2} \rho\left(\mathbf{r}_{\mathbf{c}}\right)$ indicates that there is a concentration of electronic charge at the $\mathrm{BCP}$, which indicates the covalent nature of the bond. The molecular graphs of water, ethane, cyclopropane and cubane are depicted in Figure 2 along with the 
values of $\rho\left(\mathbf{r}_{\mathbf{c}}\right)$ and $\nabla^{2} \rho\left(\mathbf{r}_{\mathbf{c}}\right)$. These values are in agreement with the standard values stipulated for covalent interaction between two atoms. Both high value of ED and the negative value of $\nabla^{2} \rho\left(\mathbf{r}_{\mathbf{c}}\right)$ characterize the covalent bonds in these chosen model systems.

\subsection{Characterization of weak interaction}

The theory of QTAIM provides an elegant approach to unravel the intermolecular interactions with the help of $\rho\left(\mathbf{r}_{\mathbf{c}}\right)$ and $\nabla^{2} \rho\left(\mathbf{r}_{\mathbf{c}}\right) .{ }^{24,27,40}$ Various classes of interactions include weak van der Waals and hydrogen bonding interactions. For these interactions, $\rho\left(\mathbf{r}_{\mathbf{c}}\right)$ is quite small $\left(\sim 10^{-2}\right.$ a.u. or less for H-bonded complexes and $10^{-3}$ a.u. for van der Waals complexes) and $\nabla^{2} \rho\left(\mathbf{r}_{\mathbf{c}}\right)$ is positive. To illustrate the power of the QTAIM, we have considered a few examples for weak van der Waals, hydrogen bonded and ionic interactions. The geometries of all these inter-molecular complexes were optimized using MP2/6-311++ $\mathrm{G}^{* *}$ level of theory. ${ }^{41}$ The interaction energies (IEs) of these complexes were calculated using supermolecule approach at MP4/6$311++\mathrm{G}^{* *}$ level ${ }^{42}$ using the following equation.

$$
\Delta E_{\text {int }}(A B)=E_{A B}^{A B}-E_{A}^{A B}-E_{B}^{A B}
$$

where, $E_{A B}^{A B}$ is the total energy of the complex $\mathrm{AB}$, $E_{A}^{A B}$ is the total energy of the monomer A calculated using the dimer basis set, $E_{B}^{A B}$ is the total energy of the monomer $\mathrm{B}$ calculated using the dimer basis set and $\Delta E_{\text {int }}(A B)$ is the IE of the complex $\mathrm{AB}$. The IEs were corrected for Basis Set Superposition Error (BSSE) employing counterpoise method. All electronic structure calculations were performed using GAUSSIAN 09 package. ${ }^{43}$ The wave function generated from these calculations were provided as the input for the QTAIM analysis. The QTAIM analysis was carried out employing AIM2000 ${ }^{44}$ and Multiwfn ${ }^{45}$ software packages.

To demonstrate the power of QTAIM in describing weak van der Waals interaction, benzene dimer has been chosen as one of the examples. Several electronic structure calculations and experimental studies ${ }^{46,47}$ have been performed on the benzene dimer to model $\pi-\pi$ interaction in biological systems. ${ }^{48-50}$ Despite several intensive efforts on this system, it was difficult to obtain the definitive structure of the benzene dimer. The role of $\mathrm{C}-\mathrm{H} . \ldots \pi$ and $\pi \ldots \pi$ interactions in the stabilization of T-shaped and parallel-displaced (PD) structures has been addressed in previous investigations. ${ }^{21}$ It is now well established by theoretical and experimental investigations that benzene dimer exists in the T-shaped and PD structures. ${ }^{51,52}$ The role of electron correlation in the stabilization of these complexes has been addressed. Classically, the electrostatic quadrupole-quadrupole interaction stabilizes the T-shaped structure when compared

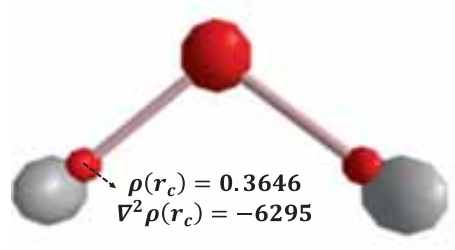

Water

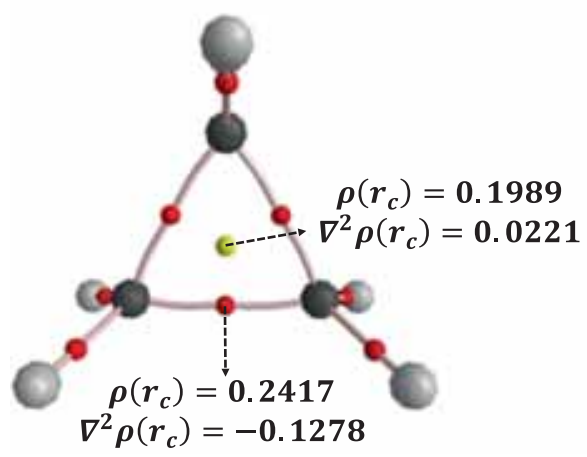

Cyclopropane

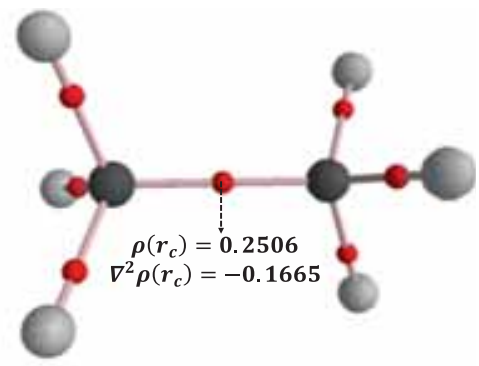

Ethane

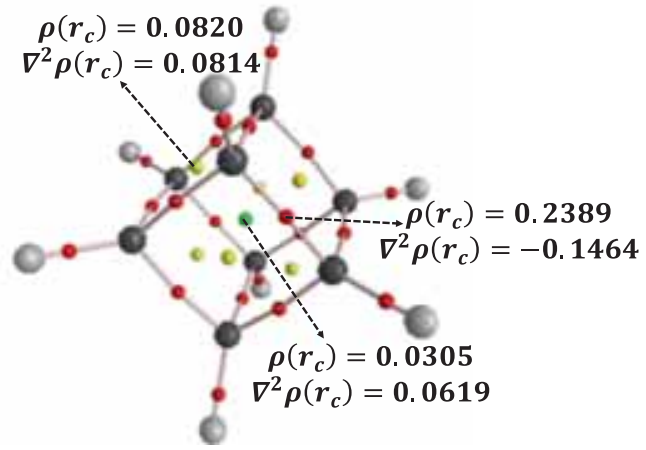

Cubane

Figure 2. Molecular graphs of water, ethane, cyclopropane and cubane systems with their electron densities and their Laplacian values in a.u. The small red, yellow and green dots represent the bond critical points, ring critical points and the cage critical points, respectively. 
to the PD structure. Hohenstein and Sherill have shown that the T-shaped and PD-structures are nearly isoenergetic. ${ }^{53}$ The optimized geometries of benzene dimer along with the molecular graphs are given in Figure 3. The calculated IEs at MP4/6-311++G** level of theory reveal that T-shaped structure $(-1.60 \mathrm{kcal} / \mathrm{mol})$ is marginally more stable than the PD-structure $(-1.20$ $\mathrm{kcal} / \mathrm{mol}$ ). The presence of $\mathrm{C}-\mathrm{H} . . \pi$ interaction in $\mathrm{T}$ shaped structure is evident from the molecular graph. The presence of RCP and CCP can be seen in the molecular graphs. The existence of weak interaction between the benzene units is evident from the values of ED and $\nabla^{2} \rho\left(\mathbf{r}_{\mathbf{c}}\right)$.

Another example considered here in the weak bonding category is the acetylene dimer. Similar to benzene, it exhibits T-shaped structure due to the presence of quadrupole-quadrupole interaction. ${ }^{54}$ The optimized geometry of acetylene dimer along with molecular graph is depicted in Figure 4. The calculated IE for acetylene dimer is $-0.93 \mathrm{kcal} / \mathrm{mol}$. The calculated $\rho\left(\mathbf{r}_{\mathbf{c}}\right)$ and $\nabla^{2} \rho\left(\mathbf{r}_{\mathbf{c}}\right)$ are 0.0078 and 0.0062 a.u., respectively. These values are akin to the range proposed in the criteria for the characterization of weak interactions. ${ }^{39}$ According to the criteria, the $\rho\left(\mathbf{r}_{\mathbf{c}}\right)$ values at the BCP range from $0.002-0.034$ a.u. ${ }^{39}$ Similarly, the $\nabla^{2} \rho\left(\mathbf{r}_{\mathbf{c}}\right)$ values at the BCPs vary from $0.024-0.139$ a.u. ${ }^{39}$

The hydrogen bonding interaction in water clusters has been the subject of numerous experimental and theoretical investigations due to their implications in chemistry and biology. ${ }^{4,55,56}$ The prototype model for the description of hydrogen bonding interaction is water dimer. Therefore, water dimer has been chosen as
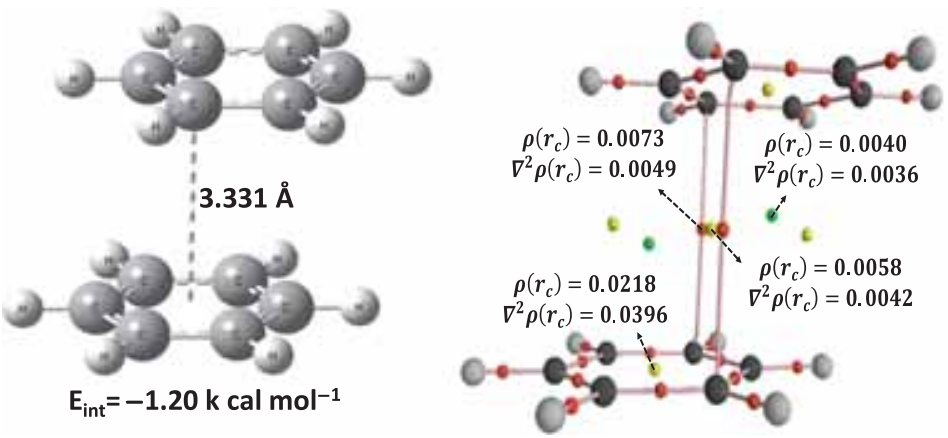

Parallel Stacked

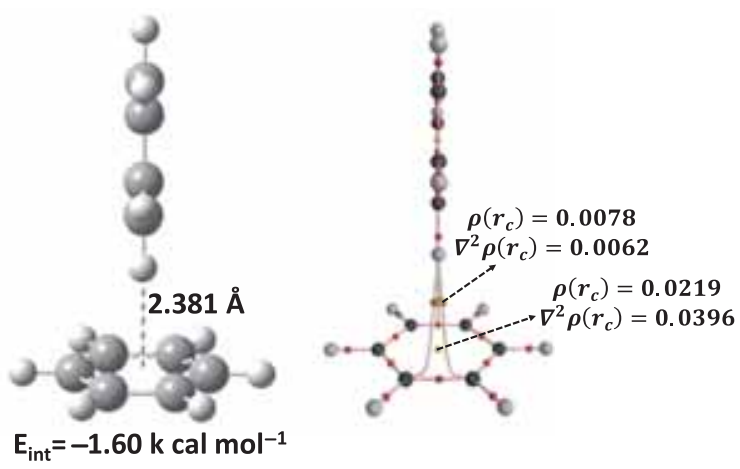

T-Shaped

Figure 3. Optimized geometries and molecular graphs of benzene dimers (T-Shaped \& Parallel Displaced) at MP2/6$311++\mathrm{G}^{* *}$ level of theory. The electron density and Laplacian values are in a.u. The small red, yellow and green dots represent the bond critical points, ring critical points and the cage critical points, respectively. The IEs were calculated at MP4/6-311++G** level of theory.
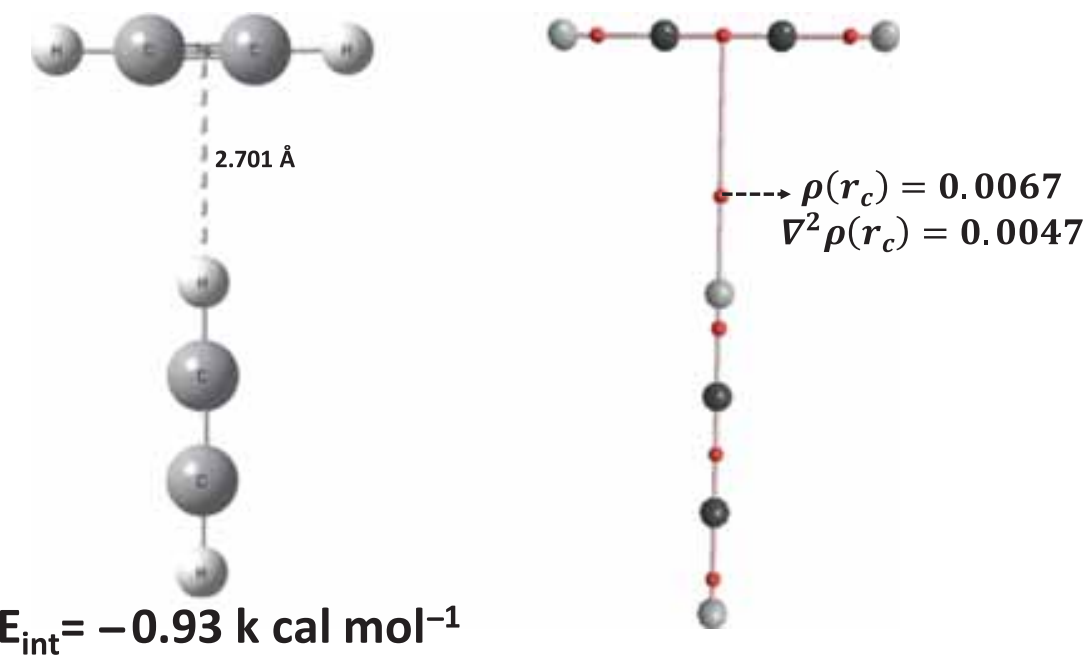

Figure 4. Optimized geometry and molecular graph of acetylene dimer at MP2/6-311++G** level of theory. The electron density and its Laplacian values are in a.u. The small red dots represent the bond critical points. The IE is calculated at the MP4/6-311++G** level of theory. 
the model system to describe hydrogen-bonding interaction with help of the QTAIM. The optimized geometry of water dimer along with the ED contour map, relief map and molecular graph are given in Figure 5.

It is evident from the ED topological parameters that $\rho\left(\mathbf{r}_{\mathbf{c}}\right)$ at the HBCP is higher than the values obtained for the benzene and acetylene dimers. It can be noticed that the $\rho\left(\mathbf{r}_{\mathbf{c}}\right)$ at the BCP increases when we move from weak van der Waals interaction to moderately strong hydrogen bonding interaction. In this category, we have also undertaken QTAIM analysis on formic acid and acetic acid dimers. The calculated IEs of
$(\mathrm{HCOOH})_{2}$ and $\left(\mathrm{CH}_{3} \mathrm{COOH}\right)_{2}$ dimers are -13.39 and $-14.40 \mathrm{kcal} / \mathrm{mol}$, respectively. The optimized geometries and molecular graphs of these dimers are shown in Figure 6. It is evident from the molecular graph that there is a $\mathrm{CP}$ between hydrogen (oxygen) of one $\mathrm{HCOOH}\left(\mathrm{CH}_{3} \mathrm{COOH}\right)$ and oxygen (hydrogen) of other $\mathrm{HCOOH}\left(\mathrm{CH}_{3} \mathrm{COOH}\right)$. Thus, the presence of donor acceptor interaction in hydrogen-bonded complexes can be clearly seen from the molecular graphs and associated topological parameters.

It is evident from the previous reports that QTAIM is highly useful to characterize the ionic molecular
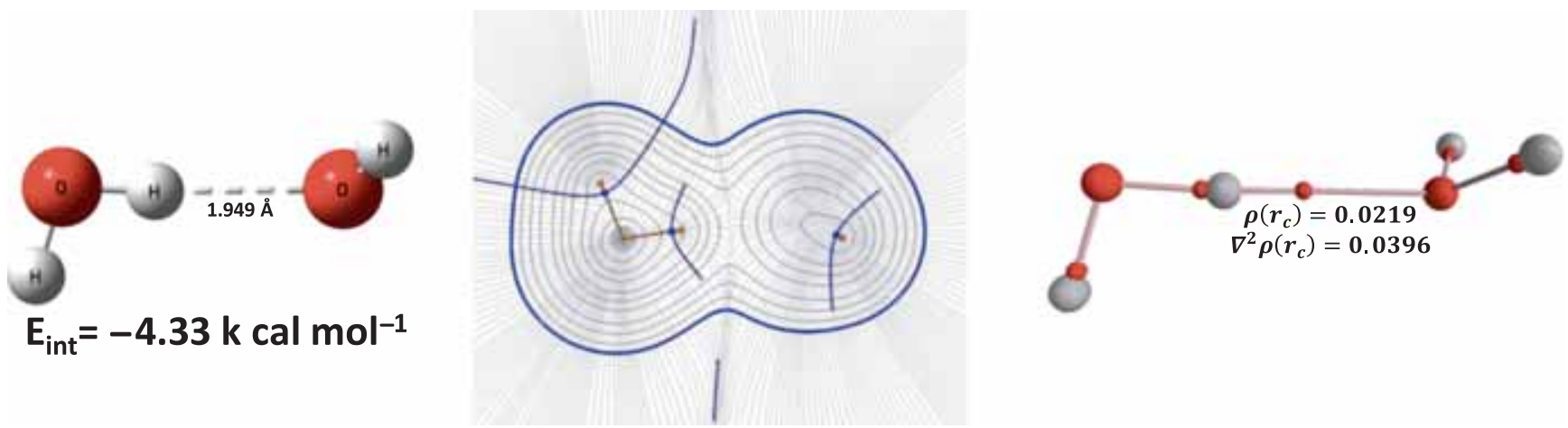

Figure 5. Optimized geometry, molecular graph and electron density contour of water dimer at MP2/6-311++G** level of theory. The electron density and its Laplacian values are in a.u. The small red dots represent the bond critical points. The IE is calculated at the MP4/6-311++ $\mathrm{G}^{* *}$ level of theory.
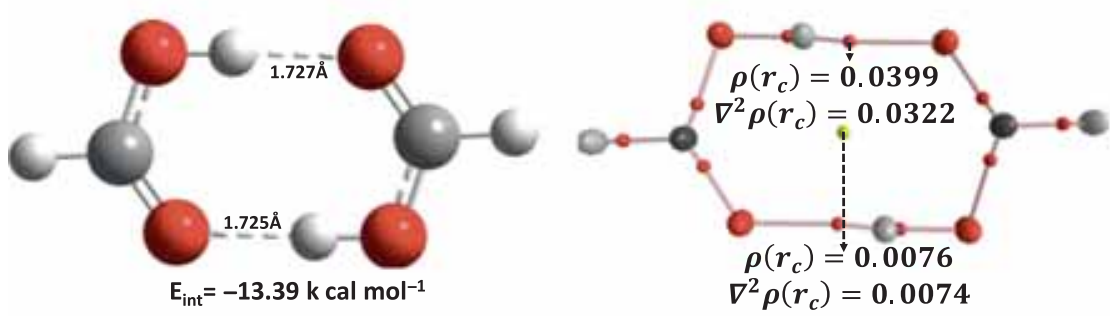

$\mathrm{HCOOH}$ dimer
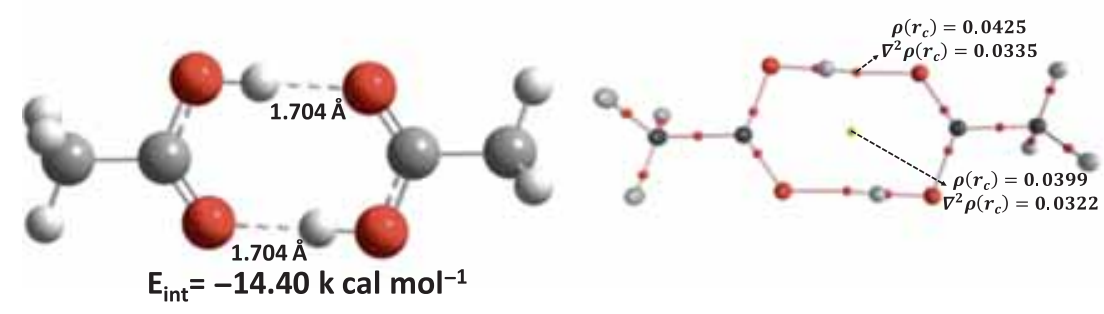

\section{$\mathrm{CH}_{3} \mathrm{COOH}$ dimer}
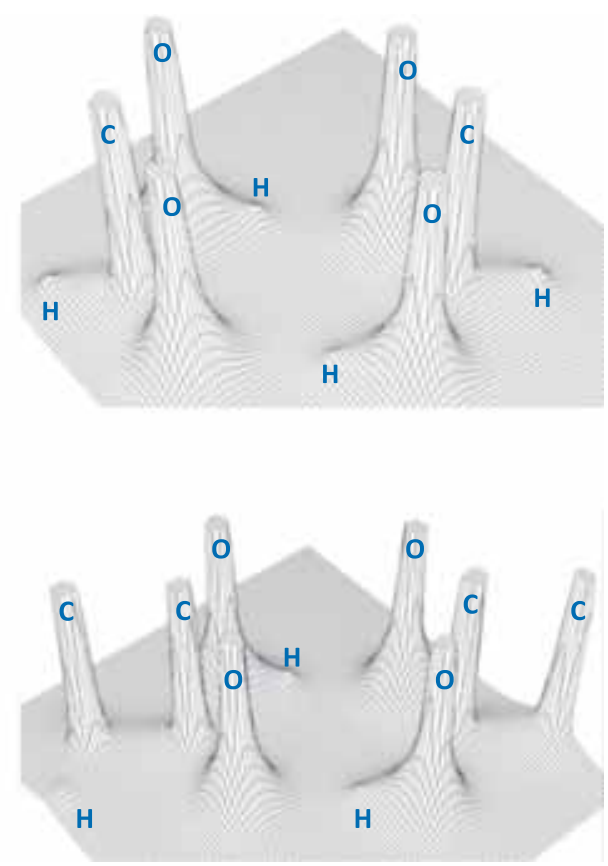

Figure 6. Optimized geometries, molecular graphs and relief maps of formic acid and acetic acid dimmers, respectively at MP2/6-311++G** level of theory. The electron densities and their Laplacian values are in a.u. The small red and yellow dots in molecular graphs represent the bond critical points and ring critical points, respectively. The IEs were calculated at MP4/6-311++G** level of theory. 
clusters. ${ }^{3,30}$ The strength of ionic hydrogen bonds ranges from $\sim 5.0$ to $35.0 \mathrm{kcal} / \mathrm{mol}$. These interactions are implicated in ionic crystals and clusters, ion solvation, electrolytes and acid-base chemistry. The importance of this interaction in proton solvation, surface phenomenon, self-assembly process in supramolecular chemistry and bio-molecular structure and function has also been recognized. The importance of charge-assisted hydrogen bonds in these systems has been addressed in previous studies. ${ }^{55-58}$ The formation of ionic hydrogen bonds involves partial proton transfer from donor to the acceptor. When a proton interacts with a single water molecule, it forms a strong covalent bond with the oxygen to form the hydronium ion $\left(\mathrm{H}_{3} \mathrm{O}^{+}\right)$, known as Eigen cation. It is a key species in the transfer of proton between molecules in the aqueous acid base conditions. Thus, the QTAIM theory has been applied to characterize the hydrogen bonding interaction in the step-wise solvation of hydronium ion. ${ }^{55}$ The optimized geometries and molecular graphs of $\mathrm{H}_{3} \mathrm{O}^{+} \ldots$ Wn
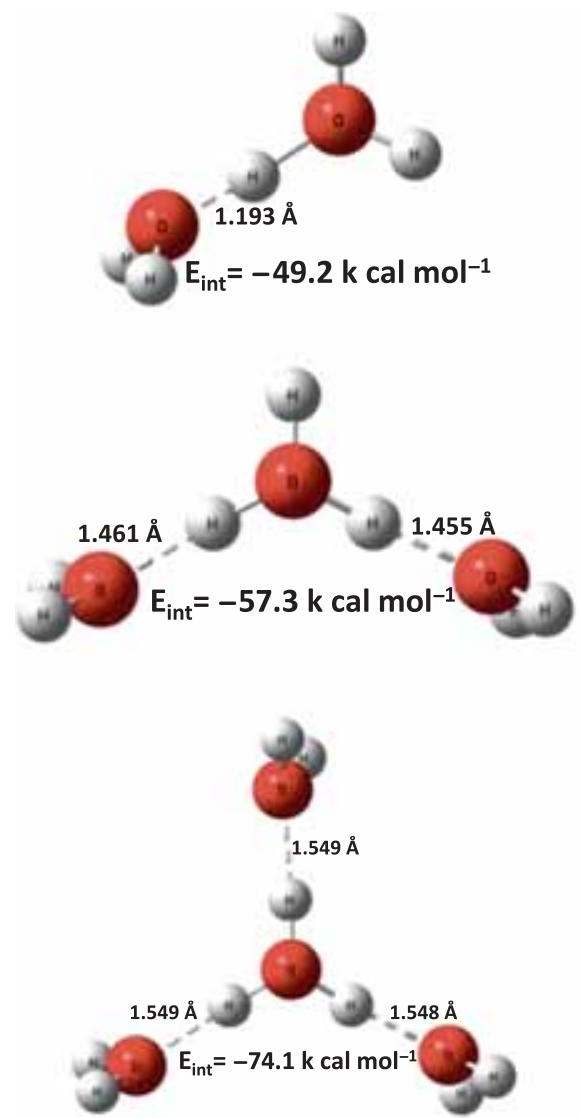

$\mathrm{H}_{3} \mathrm{O}^{+} \ldots \mathrm{W} 2$

\section{$\mathrm{H}_{3} \mathrm{O}^{+} \ldots \mathrm{W3}$}

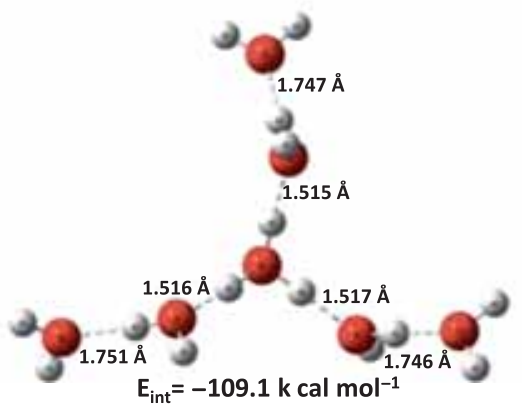

$\mathrm{H}_{3} \mathrm{O}^{+} \ldots \mathrm{W} 1$
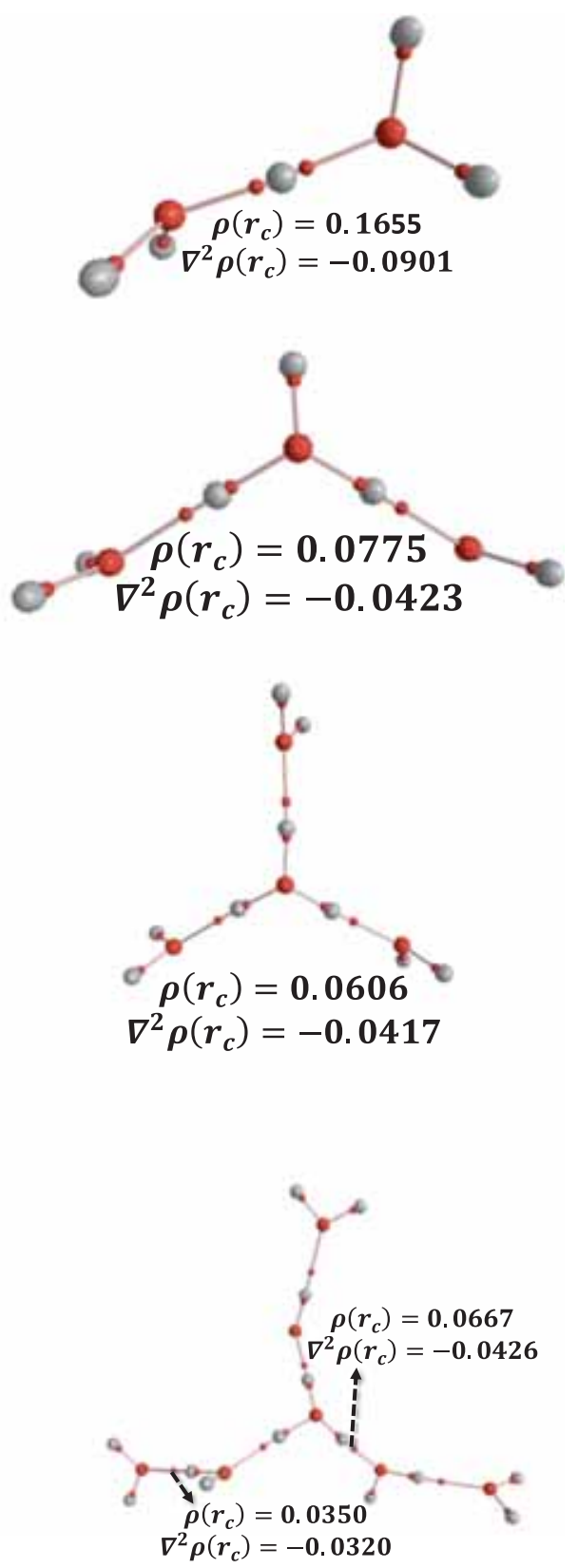

$\mathrm{H}_{3} \mathrm{O}^{+} \ldots \mathrm{W6}$

Figure 7. Optimized geometries and molecular graphs of protonated water clusters at MP2/6-311++ $\mathrm{G}^{* *}$ level of theory. The electron density and Laplacian values are in a.u. The small red dots represent the bond critical points. The IEs were calculated at MP4/6-311++G** level of theory. 
Table 1. The interaction energies (IE) of protonated water clusters along with their $\mathrm{H}$-bond distances calculated at MP4/6-311++ $\mathrm{G}^{* *}$ level of theory.

\begin{tabular}{lccc}
\hline Cluster & $\begin{array}{c}\text { Number of H-bonds } \\
\text { (distances in } \AA \text { ) }\end{array}$ & $\begin{array}{c}\text { IE } \\
(\mathrm{kcal} / \mathrm{mol})\end{array}$ & $\begin{array}{c}\text { IE (kcal/mol)/ } \\
\text { H-bond }\end{array}$ \\
\hline $\mathrm{H}_{3} \mathrm{O}^{+} \ldots \mathrm{W} 1$ & $1(1.2)$ & -49.2 & -49.2 \\
$\mathrm{H}_{3} \mathrm{O}^{+} \ldots \mathrm{W} 2$ & $2(1.5)$ & -57.3 & -28.7 \\
$\mathrm{H}_{3} \mathrm{O}^{+} \ldots \mathrm{W} 3$ & $3(1.5)$ & -74.1 & -24.7 \\
$\mathrm{H}_{3} \mathrm{O}^{+} \ldots \mathrm{W} 6$ & $6(1.5-1.7)$ & -109.1 & -18.2 \\
\hline
\end{tabular}

$(n=1,2,3,6)$ clusters are shown in Figure 7 . The calculated ED and its $\nabla^{2} \rho\left(\mathbf{r}_{\mathbf{c}}\right)$ at HBCPs for various clusters are collected in Table 1.

The value of $\rho\left(\mathbf{r}_{\mathbf{c}}\right)$ for $\mathrm{H}_{3} \mathrm{O}^{+} \ldots \mathrm{W} 1$ is 0.16 a.u. The addition of the second water molecule results in a sudden decrease in the ED values at the HBCP. Marginal changes in $\rho\left(\mathbf{r}_{\mathbf{c}}\right)$ can be noticed from the values shown in Figure 7 for the addition of the third water molecule to the hydronium ion. To predict the strength of the $\mathrm{H}$-bond formed in the second solvation shell, three more water molecules are added to form the structure $\mathrm{H}_{3} \mathrm{O}^{+} \ldots$ W6 as depicted in Figure 7. The calculated $\rho\left(\mathbf{r}_{\mathbf{c}}\right)$ values for the second solvation shell $\mathrm{H}$-bonds are found to be of the order of 0.03 a.u., which is approximately half of what has been observed for the first hydration shell. It is interesting to note that the $\nabla^{2} \rho\left(\mathbf{r}_{\mathbf{c}}\right)$ at the HBCP is negative for the $\mathrm{H}_{3} \mathrm{O}^{+} \ldots \mathrm{W} 1$ cluster, indicating the covalent character of the bond.

For other H-bonds, the $\nabla^{2} \rho\left(\mathbf{r}_{\mathbf{c}}\right)$ is positive, implying the presence of conational hydrogen bonding interaction. The strength of the first $\mathrm{H}$-bond formed in $\mathrm{H}_{3} \mathrm{O}^{+} \ldots \mathrm{W} 1$ is $\sim 50.0 \mathrm{kcal} / \mathrm{mol}$, which is in accordance with its covalent character. The SE per H-bond in $\mathrm{H}_{3} \mathrm{O}^{+} \ldots$ W2 is $\sim 29.0 \mathrm{kcal} / \mathrm{mol}$, in agreement with the decrease in the corresponding $\rho\left(\mathbf{r}_{\mathbf{c}}\right)$ values. For the completed first solvation shell structure $\mathrm{H}_{3} \mathrm{O}^{+} \ldots \mathrm{W} 3$, SE per H-bond is $\sim 25.0 \mathrm{kcal} / \mathrm{mol}$, and this is in accordance with the corresponding decrease in the $\rho\left(\mathbf{r}_{\mathbf{c}}\right)$ values. SE per $\mathrm{H}$-bond in the second solvation shell is $\sim 19.0 \mathrm{kcal} / \mathrm{mol}$, which is reflected in the decrease in the $\rho\left(\mathbf{r}_{\mathbf{c}}\right)$ value at various HBCPs. These results demonstrate that $\rho\left(\mathbf{r}_{\mathbf{c}}\right)$ and $\nabla^{2} \rho\left(\mathbf{r}_{\mathbf{c}}\right)$ values at the HBCP provide meaningful information to characterize hydrogen-bonding interactions in different solvation shells. It can also be seen that $\nabla^{2} \rho\left(\mathbf{r}_{\mathbf{c}}\right)$ is a useful parameter to differentiate conventional hydrogen bonding vs. covalent bonding in molecular clusters.

\section{Summary}

In this perspective, the basic theory and applications of quantum theory of atoms in molecules have been presented. It is evident from the results on model systems that QTAIM is an immensely useful tool to characterize the nature of bonding. In addition to the correct topological pattern of ED, the integrated properties of atoms in molecules provide further finer details on the bonding.

\section{Acknowledgements}

Authors would like to thank the Council of Scientific and Industrial Research (CSIR), New Delhi and Department of Science and Technology, India for the funding.

\section{References}

1. Bohórquez H J, Boyd R J and Matta C F 2011 J. Phys. Chem. A 11512991

2. (a) Bader R F W 1990 In Atoms in Molecules: A Quantum Theory (Oxford: Clarendon Press); (b) Bader R F W 1985 Acc. Chem. Res.18 9; (c) Bader R F W 1991 Chem. Rev. 91893

3. (a) Grabowski S J 2011 Chem. Rev. 111 2597; (b) Grabowski S J 2012 J. Phys. Chem. A 1161838

4. Parthasarthi R, Subramanian V and Sathyamurthy N 2005 J. Phys. Chem. A 109843

5. (a) Arputharaj D S, Hathwar V R, Row T N G and Kumaradhas P 2012 Cryst. Growth Des. 12 4357; (b) Hathwar V R, Paul A V, Natarajan S and Row T N G 2011 J. Phys. Chem. A 115 12818; (c) Pavan M S, Pal R, Nagarajan K and Row T N G 2014 Cryst. Growth Des. 145477

6. Hirano Y, Takeda K and Miki K 2016 Nature 534281

7. (a) Parthasarathi R, Amutha R, Subramanian V, Nair B U and Ramasami T 2004 J. Phys. Chem. A 108 3817; (b) Parthasarathi R and Subramanian V 2005 Struct. Chem. 16243

8. Szabo A and Ostlund N S 1989 In Modern Quantum Chemistry: Introduction to Advanced Electronic Structure Theory (New York: Dover Publications)

9. Popelier P 2000 In Atoms in Molecules: An Introduction (New York: Prentice Hall)

10. (a) Brown E C, Bader R F W and Werstiuk N H 2009 J. Phys. Chem. A 113 3254; (b) Bone R G A and Bader R F W 1996 J. Phys. Chem. 10010892

11. (a) Bader R F W 2009 J. Phys. Chem. A 113 10391; (b) Bader R F W 2010 J. Phys. Chem. A 1147431

12. (a) Bader R F W and Essén H 1984 J. Chem. Phys. 80 1943; (b) Bader R F W and Matta C F 2001 Inorg. Chem. 405603

13. (a) Bader R F W and MacDougall P J $1985 \mathrm{~J}$. Am. Chem. Soc. 107 6788; (d) Bader R F W and Fang D-C 2005 J. Chem. Theory Comput. 1403

14. Chaudry U A and Popelier P L A 2004 J. Org. Chem. 69 233

15. Devereux M and Popelier P L A 2007 J. Phys. Chem. A 1111536

16. Yuan Y, Mills M J L, Popelier P L A and Jensen F 2014 J. Phys. Chem. A 1187876

17. Griffiths M Z and Popelier P L A 2013 J. Chem. Inf. Model. 531714 
18. Green A J and Popelier P L A 2014 J. Chem. Inf. Model. 54553

19. Harding A P, Wedge D C and Popelier P L A $2009 \mathrm{~J}$. Chem. Inf. Model. 491914

20. Matta C F, Huang L and Massa L 2011 J. Phys. Chem. A 11512451

21. (a) Gadre S R and Pundlik S S 1995 J. Am. Chem. Soc. 117 9559; (b) Kumar R M, Elango M and Subramanian V 2010 J. Phys. Chem. A 1144313

22. (a) Jemmis E D, Subramanian G, Shrivastava I H and Gadre S R 1994 J. Phys. Chem. 98 6445; (b) Rao J S, Zipse H and Sastry G N 2009 J. Phys. Chem. B 11320

23. (a) Suresh C H, Koga N and Gadre S R 2000 Organometallics 19 3008; (b) Sharma B, Rao J S and Sastry G N 2011 J. Phys. Chem. A 1151971

24. (a) Babu K, Ganesh V, Gadre S R and Ghermani N E 2004 Theor. Chem. Acc. 111 255; (b) Grabowski S J and Lipkowski P 2011 J. Phys. Chem. A 1154765

25. Lipkowski P, Grabowski S J, Robinson $\mathrm{T} \mathrm{L}$ and Leszczynski J 2004 J. Phys. Chem. A 10810865

26. Grabowski S J, Sokalski W A and Leszczynski J 2004 J. Phys. Chem. A 1085823

27. Espinosa E, Molins E and Lecomte C 1998 Chem. Phys. Lett. 285170

28. Espinosa E, Alkorta I, Elguero J and Molins E $2002 \mathrm{~J}$. Chem. Phys. 1175529

29. Grabowski S J 2001 J. Phys. Chem. A 10510739

30. Parthasarathi R and Subramanian V 2006 In Hydrogen Bonding-New Insights S J Grabowski (Ed.) (New York: Springer) p.34

31. Popelier P L A 2008 Book Review 'Molecular Aggregation. 19th IUCr Monograph on Crystallography' by A Gavezzotti Crystallogr. Rev. 1481

32. Matta C F and Boyd R J 2007 In The Quantum Theory of Atoms in Molecules: From Solid State to DNA and Drug Design (Weinheim: Wiley-VCH)

33. Bader R F W, Johnson S, Tang H T and Popelier P L A 1996 J. Phys. Chem. 10015398

34. Fradera X, Austen A M and Bader R F W 1999 J. Phys. Chem. A 103304

35. Ziolkowski M, Grabowski S J and Leszczynski J 2006 J. Phys. Chem. A 1106514

36. Matta C F 2006 In Hydrogen Bonding - New Insights $\mathrm{S}$ J Grabowski (Ed.) (New York: Springer) Ch. 9

37. Grabowski S J 2006 In Hydrogen Bonding - New Insights (New York: Springer)

38. Popelier P L A 1998 J. Phys. Chem. A 1021873

39. Koch U and Popelier P L A 1995 J. Phys. Chem. 999747

40. Parthasarathi R, Subramanian V and Sathyamurthy N 2006 J. Phys. Chem. A 1103349

41. Møller C and Plesset M S 1934 Phys. Rev. 46618
42. Raghavachari K and Pople J A 1978 Int. J. Quantum Chem. 1491

43. Frisch M J, Trucks G W, Schlegel H B, Scuseria G E, Robb M A, Cheeseman J R, Montgomery J A Jr, Vreven T, Kudin K N, Burant J C, Millam J M, Iyengar S S, Tomasi J, Barone V, Mennucci B, Cossi M, Scalmani G, Rega N, Petersson G A, Nakatsuji H, Hada M, Ehara K, Toyota K, Fukuda R, Hasegawa J, Ishida M, Nakajima T, Honda Y, Kitao O, Nakai H, Klene M, Li X, Knox J E, Hratchian H P, Cross J B, Adamo C, Jaramillo J, Gomperts R, Stratmann R E, Yazyev O, Austin A J, Cammi R, Pomelli C, Ochterski J W, Ayala P Y, Morokuma K, Voth G A, Salvador P, Dannenberg J J, Zakrzewski V G, Dapprich S, Daniels A D, Strain M C, Farkas O, Malick D K, Rabuck A D, Raghavachari K, Foresman J B, Ortiz J V, Cui Q, Baboul A G, Clifford S, Cioslowski J, Stefanov B B, Liu G, Liashenko A, Piskorz P, Komaromi I, Martin R L, Fox D J, Keith T, Al-Laham M A, Peng C Y, Nanayakkara A, Challacombe M, Gill P M W, Johnson B, Chen W, Wong M W, Gonzalez C and Pople J A, Gaussian 09, revision A.01 (Gaussian Inc.: Wallingford CT) (2004)

44. Biegler-Konig F, Schonbohm J, Derdau R, Bayles D and Bade R F W, AIM 2000 version 1 (Bielefeld Germany) (2000)

45. Lu T and Chen F 2012 J. Comp. Chem. 33580

46. Sinnokrot O M and Sherrill C D 2004 J. Am. Chem. Soc. 1267690

47. Sinnokrot O M and Sherrill C D 2006 J. Phys. Chem. A 11010656

48. Hobza P and Havlas Z 2000 Chem. Rev. 1004253

49. Pitonak M, Neogrady P, Rezac J, Jurecka P, Urban M and Hobza P 2008 J. Chem. Theory Comput. 41829

50. Muller-Dethlefs K and Hobza P 2000 Chem. Rev. 100 143

51. Selzle H L, Schlag E W and Hobza P 1996 J. Phys. Chem. 10018790

52. Mishra B K, Arey J S and Sathyamurthy N 2010 J. Phys. Chem. A 1149606

53. Hohenstein E G and Sherrill C D 2009 J. Phys. Chem. A 113878

54. Shuler K and Dykstra C E 2000 J. Phys. Chem. A 104 4562

55. Parthasarathi R, Subramanian V and Sathyamurthy $\mathrm{N}$ 2007 J. Phys. Chem. A 11113287

56. Prakash M, Gopal Samy K and Subramanian V 2009 J. Phys. Chem. A 11313845

57. Bader R F W and Bayles D 2000 J. Phys. Chem. A 104 5579

58. Mohan N and Suresh C H 2014 J. Phys. Chem. A 118 1697 